\title{
EFFECT OF MULTIPATHS ON N-ARY TIME REVERSAL CLASSIFIERS
}

\author{
Foroohar Foroozan and Amir Asif \\ Computer Science and Engineering \\ York University, Toronto, ON, Canada \\ Tel. No.: (416) 736-2100X70128 \\ Email: $\{$ foroozan, asif\}@cse.yorku.ca
}

\begin{abstract}
The paper derives time reversal (TR) algorithms for $N$-ary classification problems and studies the impact of multipaths on the performance characteristics of these algorithms. The TR classifiers have the ability of detecting the presence or absence of a target in a rich cluttering environment with multipaths and of further classifying the detected targets either as an unknown enemy target or one of the known friendly targets. Compared to the conventional classifiers, the proposed TR classifier provides a gain of over $5 \mathrm{~dB}$ at low SNRs. An analytical multipath model, based on geometric optics approximation with strong total internal reflection from the boundaries of the medium, is used to compare the effect of multipaths on the proposed classifiers versus the conventional algorithms.
\end{abstract}

Index Terms - Time Reversal, Detection, Neyman Pearson test, Maximum Likelihood Function, and Multipaths.

\section{INTRODUCTION}

Compared with radio telecommunications, signal processing in underwater acoustics is more challenging because of lower bandwidth, high clutter, random channel characteristics, and significant distortions due to Doppler shifts and other spreading effects. In addition, the low speed of sound causes multipath propagation to stretch over time delay intervals of tens of milliseconds, which further affects the performance of the signal processing algorithms. By using the equivalent of an embedded phase conjugation equalization technique, TR [1]- [3] compensates for distortion introduced by multipaths, reduces the impact of the Doppler phenomena, and mitigates the need for a priori knowledge of the channel characteristics.

The paper derives TR algorithms for $N$-ary classification problems, which are capable of detecting the presence or absence of targets in a rich cluttering environment with multipaths and random velocity profiles. The detected target is

\footnotetext{
This work was supported in part by the Natural Science and Engineering Research Council (NSERC), Canada under Grant No. 228415-2007.
}

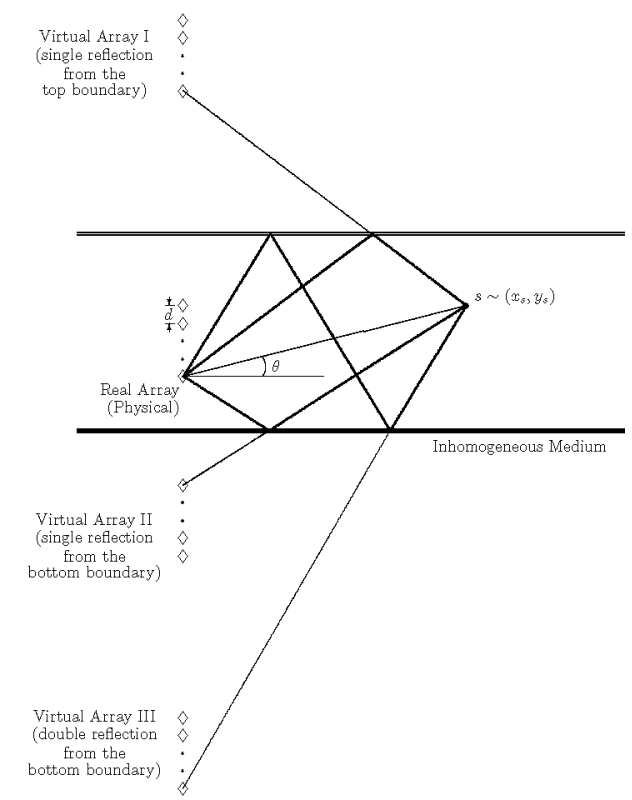

Fig. 1. Analytical multipath model used to quantify the effect of multipaths on time reversal.

further classified as an unknown enemy target or one of the known friendly targets. No prior information is assumed to be known about the enemy target, i.e., it is assumed passive with an unknown signature. Our classification algorithms use the principle of TR, where an antenna array illuminates the acoustic medium with a broadband probing signal. The backscatter of the signal is observed, time-reversed, energy normalized, and retransmitted into the medium. The TR classifier uses the reflections of the time reversed signal in the classification algorithms. The TR classifier uses the reciprocity theorem [4] such that the position of a point source and observation site can be reversed without altering the observation itself even in a lossless, inhomogeneous medium.

A second contribution of the paper is an analytical multipath framework for studying the effect of multipaths on conventional and TR classifiers in a simulated computational environment. The framework is based on the analytical mul- 
tipath model [5], illustrated in Fig. 1, which assumes strong total internal reflections from the boundary of the medium according to the geometric optics approximation. Each reflective multipath, considered in Fig. 1, is treated to originate from an additional virtual array outside the boundaries of the medium under consideration. Richer multipaths with $L$ reflections, therefore, lead to an increased number of $(2 L+1)$ arrays. Of these $(2 L+1)$ arrays, $2 L$ arrays are virtual and lie outside the domain boundaries. In array processing terminology, the TR array behaves like a lens with an effective aperture that is $(2 L+1)$ folds larger than that of the aperture of the physical array. Increasing the number of multipaths magnifies the aperture, thereby, enhancing the achievable superresolution focusing associated with TR. In comparing the effect of multipaths on TR classifier with that of the conventional classifier that uses only the forward propagation path, the final observations of both the conventional and TR classifies are normalized to have unit energy before being used by the respective detectors. esis:

Our $N$-ary classifiers are based on the following hypoth-

$$
\begin{aligned}
\mathbb{H}_{0}: & \text { No target is present. } \\
\mathbb{H}_{i}: & \text { Friendly target } i,(1 \leq i \leq(N-2)) \text {, is present. } \\
\mathbb{H}_{N-1}: & \text { Enemy target, } i=(N-1), \text { is present. }
\end{aligned}
$$

for which the the conventional and TR classifiers are presented in Section 2. The analytical multipath model is described in Section 3 and the effect of multipaths on the conventional and TR classifiers is compared through experiments in Sections 4. Finally, Section 5 concludes the paper.

\section{N-ARY MAXIMUM LIKELIHOOD CLASSIFIERS}

In this section, we introduce the notation used in the paper and present the $N$-ary maximum likelihood conventional and TR classifiers. During forward propagation, a single transceiver located at $\vec{x}_{s}=\left(x_{s}, y_{s}\right)$ probes the channel with a wideband signal $F\left(\omega_{q}\right)$, where $\omega_{q}$ denotes the discretized frequency and equals $2 q \pi / Q$, for $(0 \leq q \leq Q-1)$. The channel is probed multiple ( $1 \leq m \leq M$ ) times such that the $m$ 'th recording of the backscattered signal at the transceiver from a target at $\vec{x}_{p}=\left(x_{p}, y_{p}\right)$ is given by

$$
\begin{aligned}
& \forall q=0, \cdots, Q-1 \text { and } m=1, \cdots, M, \\
& \quad R_{i}^{(m)}\left(\omega_{q}\right)=F\left(\omega_{q}\right)\left(H_{t_{i}}\left(\omega_{q}\right)+H_{c}\left(\omega_{q}\right)\right)+V_{i}^{(m)}\left(\omega_{q}\right)(1)
\end{aligned}
$$

where $H_{c}\left(\omega_{q}\right)$ and $H_{t_{i}}\left(\omega_{q}\right)$ are, respectively, the frequency responses of the clutter and target $i . V_{i}^{(m)}\left(\omega_{q}\right)$ models zero mean, circular white Gaussian observation noise with variance $\sigma_{v}^{2}$. The probability density function (pdf) of the noise is denoted as $V_{i}^{(m)} \sim \mathcal{C N}\left(0, \sigma_{v}^{2}\right)$. We use the background subtraction process [7] to subtract clutter $F\left(\omega_{q}\right) H_{c}\left(\omega_{q}\right)$ from the recorded signal such that the processed signal is given by

$$
\begin{aligned}
& \forall q=0, \cdots, Q-1 \text { and } m=1, \cdots, M, \\
& G_{i}^{(m)}\left(\omega_{q}\right)=F\left(\omega_{q}\right) H_{t_{i}}\left(\omega_{q}\right)+V_{i}^{(m)}\left(\omega_{q}\right)
\end{aligned}
$$

Following the principle of TR, the recorded signal $G_{i}^{(m)}\left(\omega_{q}\right)$ is energy normalized, time reversed (equivalent to phase conjugation in the frequency domain), and retransmitted back into the medium. After subtraction of clutter, the backscattered TR signal at the transceiver is given by

$$
\begin{aligned}
& \forall q=0, \cdots, Q-1 \text { and } m=1, \cdots, M \\
& \begin{aligned}
P_{i}^{(m)}\left(\omega_{q}\right) & =c_{i}^{(m)} F^{*}\left(\omega_{q}\right)\left|H_{t_{i}}\left(\omega_{q}\right)\right|^{2} \\
& +c_{i}^{(m)} H_{t_{i}}\left(\omega_{q}\right) V_{i}^{(m)^{*}}\left(\omega_{q}\right)+W_{i}^{(m)}\left(\omega_{q}\right)
\end{aligned}
\end{aligned}
$$

where $W_{i}^{(m)}\left(\omega_{q}\right) \sim \mathcal{C N}\left(0, \sigma_{w}^{2}\right)$ denotes the observation noise. Stacking $G_{i}^{(m)}\left(\omega_{q}\right), P_{i}^{(m)}\left(\omega_{q}\right), H_{t_{i}}\left(\omega_{q}\right), V_{i}^{(m)}\left(\omega_{q}\right)$ and $W_{i}^{(m)}\left(\omega_{q}\right)$ for all discredited frequencies $\omega_{q}$, for $(0 \leq$ $q \leq(Q-1)$ ), respectively, in $\mathbf{G}_{i}^{(m)}, \mathbf{P}_{i}^{(m)}, \mathbf{H}_{t_{i}}, \mathbf{V}_{i}^{(m)}$ and $\mathbf{W}_{i}^{(m)}$, Eqs. (2) and (3) are expressed as

$$
\begin{aligned}
\mathbf{G}_{i}^{(m)} & =\mathbf{F H}_{t_{i}}+\mathbf{V}_{i}^{(m)} \\
\mathbf{P}_{i}^{(m)} & =c_{i}^{(m)} \mathbf{G}_{i}^{(m)^{*}} \odot \mathbf{H}_{t_{i}}+\mathbf{W}_{i}^{(m)}
\end{aligned}
$$

where $\mathbf{F}$ is a diagonal matrix of order $Q$ with $F\left(\omega_{q}\right)$ arranged along its diagonal and symbol $\odot$ denotes the Hadamard product. Given that $\mathbf{V}_{i}^{(m)} \sim \mathcal{C N}\left(\underline{0}, \sigma_{v}^{2} \mathbf{I}_{Q}\right)$ and $\mathbf{W}_{i}^{(m)} \sim$ $\mathcal{C N}\left(\underline{\mathbf{0}}, \sigma_{w}^{2} \mathbf{I}_{Q}\right)$, where $\mathbf{I}_{Q}$ is the identity matrix of order $Q$, the pdf's for $\mathbf{G}_{i}$ and $\mathbf{P}_{i}$ under hypotheses $\mathbb{H}_{i}$ are given by

$$
\begin{aligned}
\operatorname{Pr}\left(\mathbf{G}_{i} \mid \mathbb{H}_{i}\right) & =\prod_{m=1}^{M} \frac{1}{\pi^{Q}\left(\sigma_{v}^{2}\right)^{Q}} e^{-\frac{\left\|\mathbf{G}_{i}^{(m)}-\mathbf{F H}_{t_{i}}\right\|^{2}}{\sigma_{v}^{2}}} \\
\operatorname{Pr}\left(\mathbf{P}_{i} \mid \mathbb{H}_{i}\right) & =\prod_{m=1}^{M} \frac{1}{\pi^{Q}\left(\sigma_{w}^{2}\right)^{Q}} e^{-\frac{\left\|\mathbf{P}_{i}^{(m)}-c\left(\mathbf{F} \mathbf{H}_{t_{i}}\right)^{*} \odot \mathbf{H}_{t_{i}}\right\|^{2}}{\sigma_{w}^{2}}}
\end{aligned}
$$

where observations $m,(1 \leq m \leq M)$, are assumed independent of each other. For hypothesis $\mathbb{H}_{0}$, the target response $\mathbf{H}_{t_{i}}=0$. Notation $\|\cdot\|$ denotes the Frobenius norm. In Eq. (7), we have accumulated the effect of noise $v_{i}$ to $w_{i}$ in the final observation $\mathbf{P}_{i}^{(m)}$ and all normalization constants $c_{i}$ 's are assumed to be equal to $c$. Theorems 1 and 2 present the $N$-ary conventional and TR classifiers for the ideal case when target signatures $H_{t_{i}}\left(\omega_{q}\right),(1 \leq i \leq(N-1))$, are known. In general case, where the enemy target response $\mathbf{H}_{t_{N-1}}$ is unknown, Theorems 3 and 4 derive the $N$-ary conventional and TR classifiers. To save on space, proofs for Theorem 1- 4 are not included in the paper. Interested readers are referred to [6] for these proofs and further details.

Theorem 1. The test statistics $\ell_{i}\left(\mathbf{G}_{i}\right)$ of the $N$-ary conventional classifier based on the observation vector $\mathbf{G}_{i}$ is 
given by

$$
\ell_{i}\left(\mathbf{G}_{i}\right)=\Re\left(\frac{\left(\mathbf{F H}_{t_{i}}\right)^{H} \sum_{m=1}^{M} \mathbf{G}_{i}^{(m)}}{\sigma_{v}\left\|\mathbf{F H}_{t_{i}}\right\|}\right)
$$

which is linear with the following conditional pdf's.

$$
\begin{aligned}
\ell_{i}\left(\mathbf{G}_{i}\right) \mid \mathbb{H}_{0} & \sim \mathcal{C N}(0, M / 2) \\
\forall(i \neq 0), \quad \ell_{i}\left(\mathbf{G}_{i}\right) \mid \mathbb{H}_{i} & \sim \mathcal{C N}\left(\mu_{i}, M / 2\right)
\end{aligned}
$$

where mean $\mu_{i}$ equals $M\left\|\mathbf{F} \mathbf{H}_{t_{i}}\right\| / \sigma_{v}$.

Theorem 2. The linear test statistics $\ell_{i}\left(\mathbf{P}_{i}\right)$ of the $N$-ary $\mathbf{T R}$ classifier based on observation vector $\mathbf{P}_{i}$ is given by

$$
\ell_{i}\left(\mathbf{P}_{i}\right)=\Re\left(\frac{\left(\left(\mathbf{F H}_{t_{i}}\right)^{*} \odot \mathbf{H}_{t_{i}}\right)^{H} \sum_{m=1}^{M} \mathbf{P}_{i}^{(m)}}{\sigma_{w}\left\|\left(\mathbf{F} \mathbf{H}_{t_{i}}\right)^{*} \odot \mathbf{H}_{t_{i}}\right\|}\right)
$$

with conditional pdf's

$$
\begin{aligned}
\ell_{i}\left(\mathbf{P}_{i}\right) \mid \mathbb{H}_{0} & \sim \mathcal{C N}(0, M / 2) \\
\ell_{i}\left(\mathbf{P}_{i}\right) \mid \mathbb{H}_{i} & \sim \mathcal{C N}\left(\nu_{i}, M / 2\right)
\end{aligned}
$$

where $\nu_{i}$ equals $M c\left\|\left(\mathbf{F H}_{t_{i}}\right)^{*} \odot \mathbf{H}_{t_{i}}\right\| / \sigma_{w}$.

Since the effect of noise $v_{i}$ is accumulated to $w_{i}$ for the TR classifier, we note that the forward reflected signal $\mathbf{G}_{i}^{(m)}$ equals $\mathbf{F H}_{t_{i}}$.

If the channel response $\mathbf{H}_{t_{N-1}}$ for the enemy target is not known, then we use the generalized likelihood ratio (GLR)

$$
\Lambda_{N-1}^{G L R}\left(\mathbf{G}_{i}\right)=\frac{\max _{t_{N-1}}\left\{\operatorname{Pr}\left(\mathbf{G}_{i}\right) \mid \mathbb{H}_{N-1}\right\}}{\operatorname{Pr}\left(\mathbf{G}_{i} \mid \mathbb{H}_{0}\right)}
$$

in the conventional classifier. Theorem 3 presents the result.

Theorem 3. The generalized log likelihood ratio $\ell_{N-1}^{G L R}\left(\mathbf{G}_{i}\right)=$ $\ln \left(\Lambda_{N-1}^{G L R}\left(\mathbf{G}_{i}\right)\right)$ for the conventional classifier is given by

$$
\ell_{N-1}^{G L R}\left(\mathbf{G}_{i}\right) \mid \mathbb{H}_{N-1}=\sum_{q=0}^{Q-1} \frac{\left|\sum_{m=1}^{M} G_{i}^{(m)}\left(\omega_{q}\right)\right|^{2}}{M \sigma_{v}^{2} / 2}
$$

with the conditional pdf

$$
\ell_{N-1}^{G L R}\left(\mathbf{G}_{i}\right) \mid \mathbb{H}_{N-1} \sim \chi_{2 Q}^{2}(\zeta)
$$

where $\zeta=2 M \sum_{q=0}^{Q-1}\left|F\left(\omega_{q}\right)\right|^{2}\left|H_{t_{N-1}}\left(\omega_{q}\right)\right|^{2} / \sigma_{v}^{2}$. The estimate for the enemy target response is given by

$$
\widehat{H}_{t_{N-1}}=\mathbf{F}^{-1} \frac{1}{M} \sum_{m=1}^{M} \mathbf{G}_{i}^{(m)} .
$$

For the TR classifier, we stack the conjugate of the forward observations (Eq. (4)) with the TR observations (Eq. (5)) in $(2 Q M \times 1)$ vector $\mathbf{R}_{i}$ as follows

$$
\begin{aligned}
\mathbf{R}_{\mathbf{i}} & =\left[\begin{array}{c}
\mathbf{G}_{i}^{*} \\
\mathbf{P}_{i}
\end{array}\right] \\
& =\left[\begin{array}{c}
\mathbf{1} \otimes\left(\mathbf{F} \mathbf{H}_{t_{i}}\right)^{*} \\
\mathbf{c}_{\mathbf{i}} \otimes\left[\mathbf{F}^{*} \mathbf{H}_{t_{i}}^{*} \odot \mathbf{H}_{t_{i}}\right]
\end{array}\right]+\left[\begin{array}{c}
\mathbf{V}_{i}^{*} \\
\left(\mathbf{c}_{\mathbf{i}} \otimes \mathbf{H}_{t_{i}}\right) \odot \mathbf{V}_{i}^{*}+\mathbf{W}_{i}
\end{array}\right] \cdot y_{c} .
\end{aligned}
$$

In terms of $\mathbf{R}_{\mathbf{i}}$, the GLR test for the TR detector is given by

$$
\Lambda_{N-1}^{G L R}\left(\mathbf{R}_{i}\right)=\frac{\max _{t_{N-1}}\left\{\operatorname{Pr}\left(\mathbf{R}_{i} \mid \mathbb{H}_{N-1}\right)\right\}}{\operatorname{Pr}\left(\mathbf{R}_{i} \mid \mathbb{H}_{0}\right)}
$$

To save on space, we express $F\left(\omega_{q}\right)$ as $F, G_{i}\left(\omega_{q}\right)$ as $G_{i}$, and $\widehat{H}_{t_{N-1}}\left(\omega_{q}\right)$ (estimate of $\left.H_{t_{N-1}}\left(\omega_{q}\right)\right)$ as $\widehat{H}_{t_{N-1}}$ in the following theorem.

Theorem 4. The generalized log likelihood ratio test $\ell_{N-1}^{G L R}\left(\mathbf{R}_{i}\right)$ for the TR classifier is given by

$$
\begin{aligned}
& \ell_{N-1}^{G L R}\left(\mathbf{R}_{i} \mid \mathbb{H}_{N-1}\right)= \\
& \quad \frac{1}{\sigma_{v}^{2} \sigma_{w}^{2}} \sum_{m=1}^{M} \sum_{q=0}^{Q-1}\left[\left\{\sigma_{v}^{2} c_{i}^{(m)^{2}}\left|G_{i}^{(m)}\right|^{2}+\sigma_{w}^{2}|F|^{2}\right\}\left|\widehat{H}_{t_{N-1}}\right|^{2}\right. \\
& \left.\quad-2 \Re\left\{\left(c_{i}^{(m)} P_{i}^{(m)^{*}} G_{i}^{(m)^{*}} \sigma_{v}^{2}+G_{i}^{(m)^{*}} F \sigma_{w}^{2}\right) \widehat{H}_{t_{N-1}}\right\}\right]
\end{aligned}
$$

where $\widehat{H}_{t_{N-1}}$ is given by

$\widehat{H}_{t_{N-1}}=\frac{\sum_{m=1}^{M}\left[\frac{G_{i}^{(m)}\left(\omega_{q}\right) F\left(\omega_{q}\right)^{*}}{\sigma_{v}^{2}}+\frac{c_{i}^{(m)} G_{i}^{(m)}\left(\omega_{q}\right) P_{i}^{(m)^{*}}\left(\omega_{q}\right)}{\sigma_{w}^{2}}\right]}{\sum_{m=1}^{M}\left[\frac{\left|F\left(\omega_{q}\right)\right|^{2}}{\sigma_{v}^{2}}+\frac{\left|G_{i}^{(m)}\left(\omega_{q}\right)\right|^{2} c_{i}^{(m)^{2}}}{\sigma_{w}^{2}}\right]}$.

The Neyman Pearson test computes the thresholds $\eta_{i}$ associated with hypothesis $\mathbb{H}_{i}$, for $(1 \leq i \leq(N-1))$, by fixing the probability of false alarm

$$
P_{F}=\int \ldots \int_{\mathbf{Z}} \operatorname{Pr}\left(\ell_{1}, \ldots, \ell_{N-1} \mid \mathbb{H}_{0}\right) d \ell_{1} \ldots d \ell_{N}
$$

where $\mathbf{Z}=\mathbf{Z}_{1} \cup \ldots \cup \mathbf{Z}_{N-1}$ with $\mathbf{Z}_{i}$ being the observation space associated with hypothesis $\mathbb{H}_{i}$ corresponding to the presence of target $i$. For a 3 -ary detection problem, the expression for the probability of false alarm reduces to

$$
\begin{gathered}
P_{F}=\frac{3}{4}-\frac{1}{4}\left[\operatorname{erf}\left(\frac{\eta_{1}}{\sqrt{M}}\right)-\operatorname{erf}\left(\frac{\eta_{2}}{\sqrt{M}}\right)-\operatorname{erf}\left(\frac{\eta_{1}}{\sqrt{M}}\right) \operatorname{erf}\left(\frac{\eta_{2}}{\sqrt{M}}\right)\right] \\
\text { where } \quad \operatorname{erf}(x)=\frac{2}{\sqrt{\pi}} \int_{0}^{x} e^{-t^{2}} d t, \quad x \in \mathbb{R} .
\end{gathered}
$$

\section{ANALYTICAL MULTIPATH MODEL}

To model the effect of multipaths, we use the analytical model [5] that is based on the geometric optics approximation with strong total internal reflections from the boundaries of the medium. The analytical multipath model is shown in 8) Fig. 1, where the bottom boundary of the channel is assumed aligned with the $x$-axis and the positive $y$-axis is oriented in the upward direction. The width of the channel is denoted by 


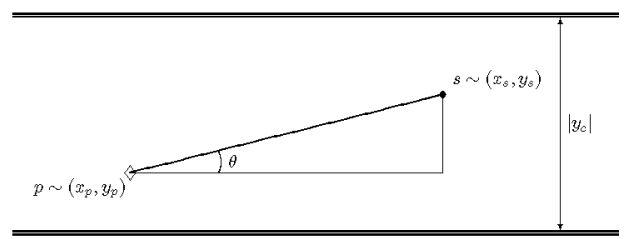

(a) Direct path.

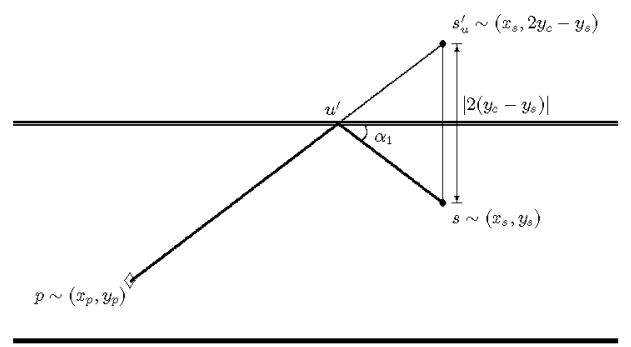

(b) Top-boundary, single reflection path.

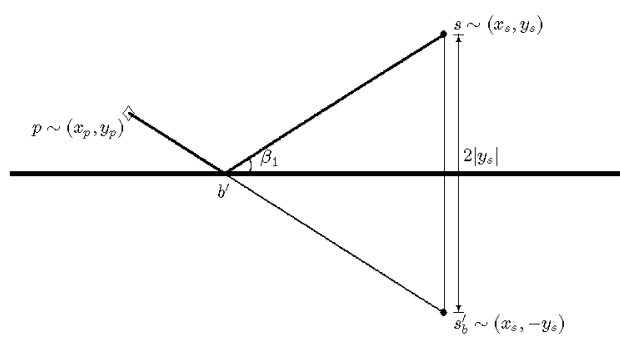

(c) Bottom-boundary, single reflection path.

Fig. 2. Multipaths between transceiver $s$ and target $p$ in TR: (a) Direct path; (b) Reflection from the top boundary; and (c) Reflection from the bottom boundary.

The transceiver at $\left(x_{s}, y_{s}\right)$ probes the channel with the wideband signal $F\left(\omega_{q}\right)$. The target located at $\left(x_{p}, y_{p}\right)$ receives several delayed and distorted versions of the probing pulse due to multipaths. These multipaths are shown in subplots (a)-(e) in Fig. 2 in terms of a single transducer element. In reality, each transducer element would be replaced by a correctly oriented antenna array.

Direct Path: models the direct propagation of $F\left(\omega_{q}\right)$ between the transceiver and the target. The direct path is shown as solid line in Fig. 2(a).

Top-Boundary, Single-Reflection Path: models the multipath taken by the signal reflected once from the top boundary. As shown in Fig. 2(b), the top-boundary single reflection path can be simulated by ignoring the boundary reflection and introducing a virtual transceiver at $\left(x_{s}, 2 y_{c}-y_{s}\right)$ above the top boundary of the channel.

Bottom-Boundary, Single-Reflection Path: is similar to the top-boundary single-reflection path except that the reflec-

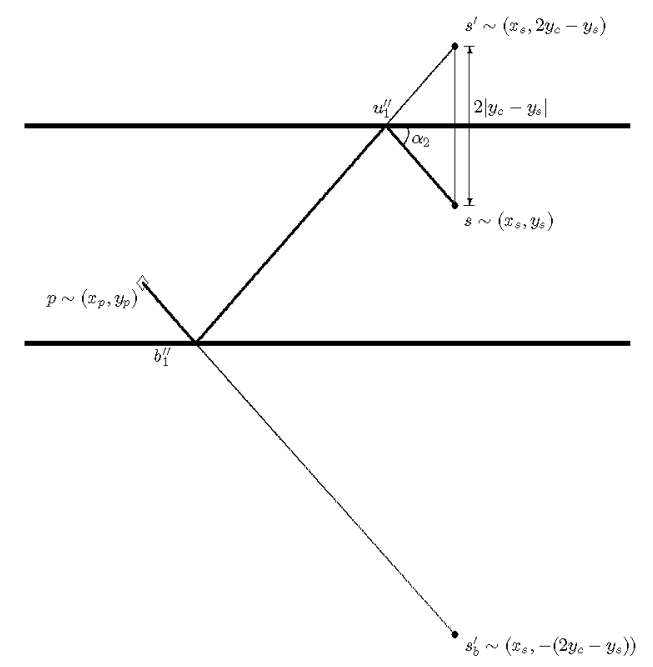

(d) Top-bottom, double reflection path.

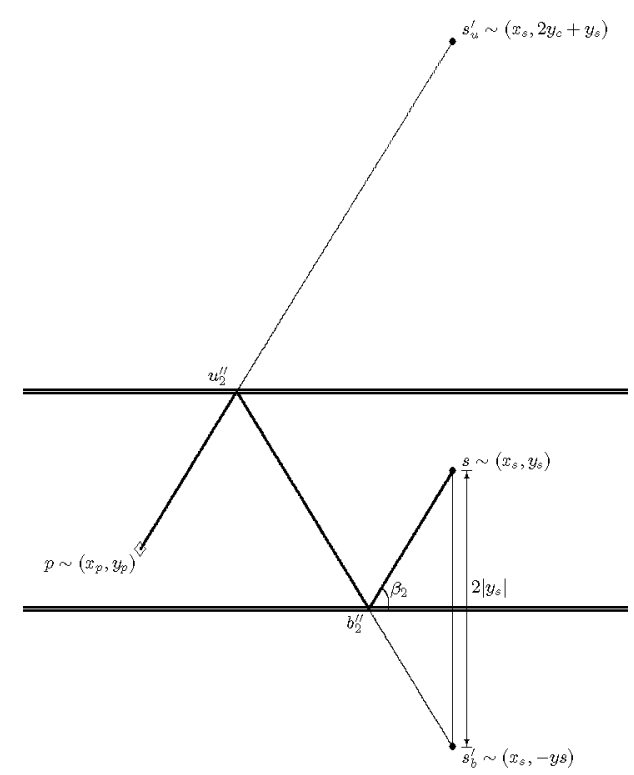

(e) Bottom-top, double reflection path.

Fig. 2. (contd.) (d) Reflection from the top boundary followed by a second reflection from the bottom boundary; and (e) Reflection from the bottom boundary followed by a second reflection from the top boundary.

tion takes place from the bottom of the channel as shown in Fig. 2(c). The bottom-boundary, single reflection path is simulated by adding a virtual transceiver at $\left(x_{s},-y_{s}\right)$ that transmits a signal directly to the target.

Top-Bottom, Double Reflection Path: is shown in terms of three bold lines in Fig. 2(d) and consists of paths joining: (i) the transceiver located at $\left(x_{s}, y_{s}\right)$ with the reflection point $u_{1}^{\prime \prime}$ at the top boundary; (ii) the top reflection point $u_{1}^{\prime \prime}$ with the reflection point $b_{1}^{\prime \prime}$ at the bottom boundary; and (iii) the bottom reflection point $b_{1}^{\prime \prime}$ with the target $\left(x_{p}, y_{p}\right)$. This is 


\begin{tabular}{|c|c|c|}
\hline \multirow{2}{*}{ Multipath } & \multicolumn{2}{|c|}{ Locations } \\
\cline { 2 - 3 } & Transceivers $(\times \lambda)$ & Targets $(\times \lambda)$ \\
\hline $\mathbf{1}$ & $(220,30)$ & $(270,10)$ \\
\hline \multirow{5}{5}{} & $(220,50),(220,-30)$ & $(270,70),(270,-10)$ \\
\hline 7 & $(220,110),(220,-50)$ & $(270,90),(270,-70)$ \\
\hline & $(220,130),(220,-110)$ & $(270,150),(270,-90)$ \\
\hline 11 & $(220,190),(220,-130)$ & $(270,170),(270,-150)$ \\
& $(220,210),(220,-190)$ & $(270,230),(270,-170)$ \\
\hline
\end{tabular}

Table 1. Locations of virtual transceivers and targets, each subsumes the respective virtual transceivers or targets for the lower order, i.e., the virtual transceivers for $(m=7)$ includes the virtual transceivers for $(m=5)$ and $(m=1)$.

simulated by a direct propagation from the virtual transceiver located at $\left(x_{s},-\left(2 y_{c}-y_{s}\right)\right)$ to the target.

Bottom-Top, Double Reflection Path: is similar to the topbottom, double reflection path except that the first reflection takes place from the bottom boundary. As shown in Fig. 2(e), the bottom-top, double reflection path is modeled by the virtual transceiver located at $\left(x_{s}, 2 y_{c}+y_{s}\right)$ that transmits directly in a straight line to the target.

The second column of Table 1 uses the aforementioned analytical model to specify the locations of the virtual array when a single transceiver located at coordinate $(220,30) \lambda$ is transmitting. The width $y_{c}$ of the channel is 40 . Therefore, only the real transceiver located at $(220,30) \lambda$ is needed to model the $(m=1)$ multipath. Notation $(m=5)$ includes the five multipaths included in subplots (a)-(e) of Fig. 2. Such a five multipath model includes the real transceiver located at $(220,30) \lambda$ as well as four virtual transceivers located at $(220,50) \lambda,(220,-30) \lambda,(220,110) \lambda$, and $(220,-50) \lambda$. Similarly, notation $(m=7)$ includes the real transceiver, the virtual transceivers for $(m=5)$ multipaths, and additional virtual transceivers located at $(220,130) \lambda$ and $(220,-110) \lambda$. The $m$ multipath propagation of the probing pulse $F\left(\omega_{q}\right)$ from the transceiver to the target is modeled by $m$ direct propagations, one from the real transceiver and the remaining $(m-1)$ from the $(m-1)$ virtual transceivers whose locations are specified in the second column of Table 1 to a single target.

The above procedure can also be used to model multipaths for the backscattered signal reflected from the target to the transceiver. The $m$ multipath propagation of the reflected signal from the target to the transceiver is again modeled by $m$ direct propagations, one from the real target and the remaining $(m-1)$ components from the $(m-1)$ virtual targets. For the real target located at $(270,10) \lambda$, the locations of the virtual targets used to model backscatter signal reflected from the target to the transceiver for different values of $m$ are listed in the third column of Table 1 .

\section{EXPERIMENTS}

A single transceiver probes the channel with pulse

$$
f(t)=-2 \pi^{2} v^{2}(t-1 / v) e^{-\pi^{2} v^{2}(t-1 / v)^{2}},
$$

for $(0 \leq t \leq 0.7 \mu \mathrm{s})$. Symbol $v$ represents the carrier frequency, which equals $3 \mathrm{MHz}$. The probing pulse is transmitted into the medium according to the wave equation

$$
\Delta \psi(\vec{y}, \vec{x}, t)-\frac{1}{(c(\vec{x}))^{2}} \frac{\partial^{2} \psi(\vec{y}, \vec{x}, t)}{\partial t^{2}}=-f(t) \delta(\vec{x}-\vec{y})
$$

where $\vec{x}$ denotes the coordinates of the observation site and $\vec{y}$ the coordinates of the probing site. $c(\vec{x})$ is the random velocity of the medium, which has a Gaussian distribution with a mean of $c_{0}$ equal to $1500 \mathrm{~m} / \mathrm{s}$ and a standard deviation of $10 \%$ about the mean. Based on the CFL conditions, the time step $\Delta t$ is set to $5 \times 10^{-8} \mathrm{~s}$.

The channel is modeled as a two dimensional domain $R$ with discretization steps $\Delta x=\Delta y=\frac{\lambda}{4}, \lambda$ being the wavelength of $0.5 \mathrm{~mm}$. The discretized channel has finite dimensions of $(0 \leq i \leq I-1)$ and $(0 \leq j \leq J-1)$, where $i, j$ are spatial indices representing site $\vec{x}=(i \Delta x, j \Delta y)$. Symbol $k$ is the time index. The wave equation in (24) is discretized using the following finite difference approximation

$$
\frac{\partial^{2} \psi}{\partial t^{2}}=\psi_{i, j, k+1}-2 \psi_{i, j, k}+\psi_{i, j, k-1}
$$

with respect to time $t$. The second order derivatives of the field $\psi$ with respect to $x$ and $y$ are discretized using similar relationships. The finite difference representation of Eq. (24) is

$$
\begin{aligned}
\psi_{i, j, k+1} & =2 \psi_{i, j, k}+k_{x}^{2}\left(\psi_{i+1, j, k}+\psi_{i-1, j, k}-2 \psi_{i, j, k}\right)(26) \\
& +k_{y}^{2}\left(\psi_{i, j+1, k}+\psi_{i, j-1, k}-2 \psi_{i, j, k}\right)-\psi_{i, j, k-1}
\end{aligned}
$$

where $k_{x}=c \Delta t / \Delta x$ and $k_{y}=c \Delta t / \Delta y$. In our simulations, the coordinates of the transceiver and target are $(220,30) \lambda$ and $(270,10) \lambda$, respectively. The width of the channel is $40 \lambda$. To quantify the effect of multipaths on time reversal, the setup introduced in Section 3 is used. In our simulations, we implement 3-ary TR and conventional classifiers with 5 , 7,11 , and 21 multipaths. The 3 -ary detection problem corresponds to determining if a target is present and further differentiating the identity of the target as friendly or enemy target. The locations of the scatterers (unwanted elements) are randomized during the simulations when the receiver operating characteristics are derived. In our experiments, we use a single transceiver as a transmitting/receiving antenna so that the TR gain is attributed only to the multipaths, the factor that we want to highlight in this paper. The locations of real and virtual transceivers and targets are specified in Table 1. We use the Neyman Pearson test with the likelihood functions specified in Theorem 1 for the conventional classifier and Theorem 2 for the TR classifier for the friendly target 


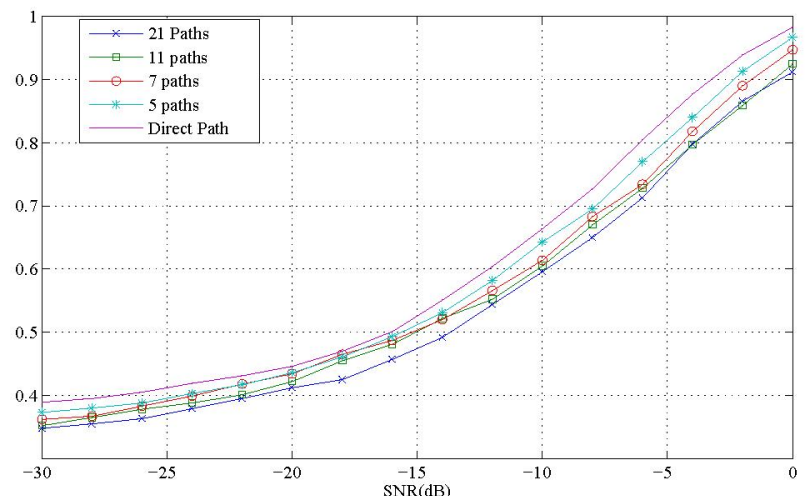

(a) Conventional Classifier.

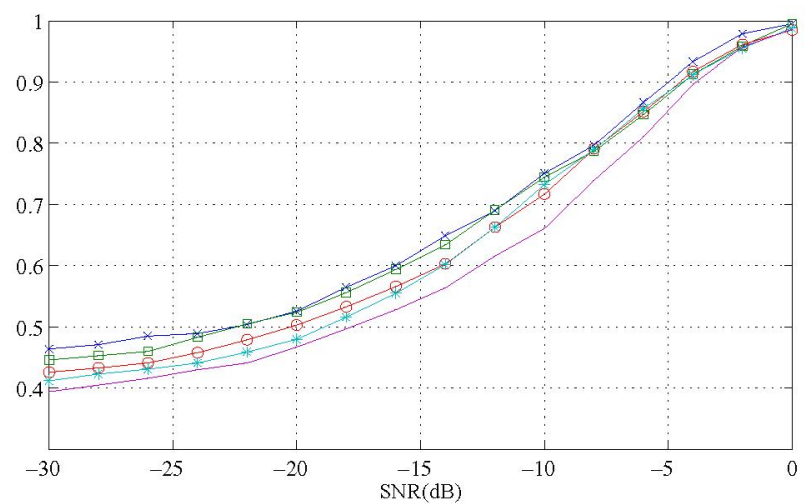

(b) TR Classifier.

Fig. 3. Probability of Detection of the enemy target versus SNRs for different multipaths: (a) Conventional classifier (b) TR classifier.

and GLR functions given in Theorem 3 for the conventional classifier and Theorem 4 for the TR classifier for the enemy target. Thresholds $\eta_{1}$ and $\eta_{2}$ in the Neyman Pearson test are computed by setting the probability of false alarm to 0.01 in Eq. (22).

Fig. 3 plots the probabilities of detection $P_{D}$ for the enemy target as a function of SNR. This figure shows the effect of multipaths on the conventional classifier (top subplot) and TR classifier (bottom subplot). With an increase in the number of multipaths, the performance of the conventional classifier deteriorates substantially. For $P_{D}=0.5$, there is a loss in performance of about $5 \mathrm{~dB}$ between the direct path $(m=1)$ versus ( $m=21)$ multipaths. On contrary to the conventional classifier, the performance of the TR classifier improves with an increase in the number of multipaths. For $P_{D}=0.5$, the $(m=21)$ multipath model provides a gain of $5 \mathrm{~dB}$ over the direct path where no multipath is considered. On average, each additional multipath provides a gain of about $0.25 \mathrm{~dB}$ in the TR classifier at low SNRs, while there is a loss of almost the same amount in the gain of the conventional classifier per multipath.

\section{CONCLUSION}

We derive the $N$-ary TR and conventional ML classifiers and present an analytical multipath model to study the effects of multipaths on our proposed classifiers. Our analytical multipath model is based on geometric optics approximation, which assumes strong total internal reflections from the boundaries of the medium. While multipaths have a negative impact on the performance of the conventional classifiers based on the backscatter of the probing signal, the performance of the TR classifier that uses backscatters of both the probing signal and the time reversed signal improves with increase in the number of multipaths. In array processing terminology, the TR array behaves like a lens with an effective aperture that is several folds larger than that of the aperture of the physical array. Increasing the number of multipaths magnifies the aperture by a similar factor, thereby, resulting in performance gains for the TR classifier of about $1 / 4 \mathrm{~dB}$ per additional multipath.

\section{REFERENCES}

[1] M. Fink, "Time Reversal Acoustics," Physics Today, vol. 39 , no. 5, 1997, pp. 555-566.

[2] L. Borcea, G. Papanicolaou, C. Tsogka, and J. Berrymen, "Imaging and Time Reversal in Random Media," Inverse Problems, vol. 18, 2002, pp. 1247-1289

[3] H. C. Song, W. A. Kuperman, W. S. Hodgkiss, T. Akal, and C. Ferla, "Iterative Time Reversal in the Ocean Reversal," J. Acoustic Society of America, vol. 105, no. 6, 1999, pp. 3176-3184.

[4] Mathias Fink, "Time reversal of Ultrasonic Fields - Part I: Basic Principles," IEEE Transactions on Ultrasonics, Ferroelectrics, and Frequency control, vol. 39, no. 5, pp. 555-566, 1992.

[5] A. Asif, Q. Bai, and J. M. F. Moura, "Time Reversal Matched Field Processing: An Analytical Justification," in Proceedings of ISSPIT, Vancouver, 2006.

[6] F. Foroozan and A. Asif, " $N$-Ary Maximum Likelihood Target Detection With Time Reversal," in Proceedings of the IEEE International Symposium on Information Theory (ISIT), Toronto, July 2008, pp. 1873-1877.

[7] J. M. F. Moura and Y. Jin, "Detection by time reversal: single antenna,"IEEE Trans. on Signal Processing, vol. 55, 2007, pp. 187-201. 